\title{
Correction to: An Exploration of the Role of Sugar-Sweetened Beverage in Promoting Obesity and Health Disparities
}

\author{
Desiree M. Sigala $^{1}$ (D) Kimber L. Stanhope $^{1,2}$ (D) \\ Published online: 2 February 2021 \\ (C) Springer Science+Business Media, LLC, part of Springer Nature 2021
}

\section{Correction to: Curr Obes Rep https://doi.org/10.1007/s13679-020-00421-x}

The original version of this article unfortunately contained some mistakes.

Reference Citations " $121,122 \bullet$, 123-127, 128••, 129, 130•, 131-133, 134••, 135, 136•, 137-149, 150•, 151-153” were added into the last sentence of the review as follows:

"Therefore, in response to question 4, we echo others $[120 \bullet \bullet, 121,122 \bullet \bullet, 123-127,128 \bullet \bullet, 129,130 \bullet, 131-$ $133,134 \bullet \bullet, 135,136 \bullet, 137-149,150 \bullet, 151-153,155 \bullet \bullet]$ in suggesting that proactive environmental measures to decrease exposure to palatable food cues, particularly in the form of targeted marketing to our vulnerable populations, is a necessary strategy for slowing the obesity epidemic."
The correct citations for this sentence were $[120 \bullet, 155 \bullet \cdot]$ to read:

\begin{abstract}
"Therefore, in response to question 4, we echo others $[120 \bullet, 155 \cdot \bullet]$ in suggesting that proactive environmental measures to decrease exposure to palatable food cues, particularly in the form of targeted marketing to our vulnerable populations, is a necessary strategy for slowing the obesity epidemic."
\end{abstract}

The original article has been corrected.

The online version of the original article can be found at https://doi.org/ 10.1007/s13679-020-00421-x

Kimber L. Stanhope

klstanhope@ucdavis.edu

Desiree M. Sigala

dmsigala@ucdavis.edu

1 Department of Molecular Biosciences, School of Veterinary Medicine, University of California (UC), Davis, 2211 VM3B, Davis, CA 95616, USA

2 Basic Sciences, Touro University of California, Vallejo, CA, USA 